\title{
REVIEW \\ Agricultural Vehicle Navigation System: Development of a Guidance Information Display
}

\author{
Yasuyuki HAMADA $^{1 *}$, Yosuke MATSUO ${ }^{1}$ and Takashi YAMASHITA ${ }^{1}$ \\ ${ }^{1}$ Fundamental Technology Department, Bio-oriented Technology Research Advancement \\ Institution, National Agriculture and Food Research Organization \\ (Saitama, Saitama 331-8537, Japan)
}

\begin{abstract}
We developed a navigation system for agricultural vehicles that displays optimum work paths and guides steering to support precision farming. It comprises navigation sensors, including a GPS receiver and an azimuth sensor, and a PC that processes the inputs and displays the information. It is operated via a game controller. The control software supports configuring functions, adjusting settings, displaying results, and recording and managing information. It has functions appropriate for Japanese agriculture, allowing ease of handling and working across multiple fields. To verify its utility, we simulated work using a tractor fitted with it. It improved both work accuracy and efficiency. We confirmed its utility and ease of handling in actual farming work. Trial sales of the navigation system were begun in 2007.
\end{abstract}

Discipline: Agricultural machinery

Additional key words: driving support device, framework, GPS, navigation software

\section{Introduction}

In farming work using agricultural vehicles such as tractors, it is important to minimize the area of unworked and double-worked land by driving the vehicle straight and at a constant interval in the field in order to achieve high efficiency and the optimum use of inputs such as fertilizers and chemicals. Various driving support devices, including light bars to display the distance and direction to the recommended work path, have been developed in Europe and North America ${ }^{1}$. These devices have proved to have limited popularity in Japan ${ }^{3}$, where (1) in many cases the vehicle must move frequently among the multiple small fields worked in Japanese agriculture, (2) the equipment and machinery are relatively small, (3) translation of the built-in software and manuals into Japanese would entail great cost, and (4) appropriate aftersales support is not provided.

Therefore, we developed a navigation system for agricultural vehicles that indicates the optimum work path and offers guidance for steering during work in the field to optimize efficiency and accuracy. The system has functions appropriate for Japanese agriculture, allowing ease of handling while moving frequently among fields.

Here we outline the navigation system and report the results of field testing.

\section{Outline of the navigation system}

\section{Composition of the navigation system}

The navigation system is composed of navigation sensors, including a Geographical Positioning System (GPS) receiver and an optional azimuth/attitude sensor, a computer to process and display sensor information (the "navigation computer"), and a game controller (Fig. 1). Any standard GPS receiver that outputs position information in NMEA-0183 format ${ }^{4}$ via RS232 or UDP protocol can be used. Furthermore, if the navigation sensors can output the attitude information together with position information, it becomes possible to compensate for the gap between the antenna position on a slope and its position in the field.

The navigation computer runs Microsoft Windows XP or Vista and navigation software. The navigation software processes information from the navigation sen-

*Corresponding author: e-mail yhamada@affrc.go.jp

Received 3 March 2008; accepted 20 August 2008. 
sors and displays the appropriate work path, peripheral map information, and current work state on the screen. The navigation software requires Microsoft .NET Framework 2.0 and Microsoft Managed DirectX 1.1 software. The operator controls the navigation system via a standard game controller.

Figure 2 depicts the operator's cabin of a tractor fitted with the navigation system.

\section{Navigation software}

\section{(1) Composition of the navigation software}

The navigation software is composed of an internal operation processor and a user interface.

The internal operation processor uses a multi-purpose library called the framework, which handles basic common operations such as configuring functions, adjusting settings, displaying results, recording and managing information ${ }^{2}$, setting the work width and recommended work path, and calculating deviations from the path. Other functions represent the vehicle and the navigation

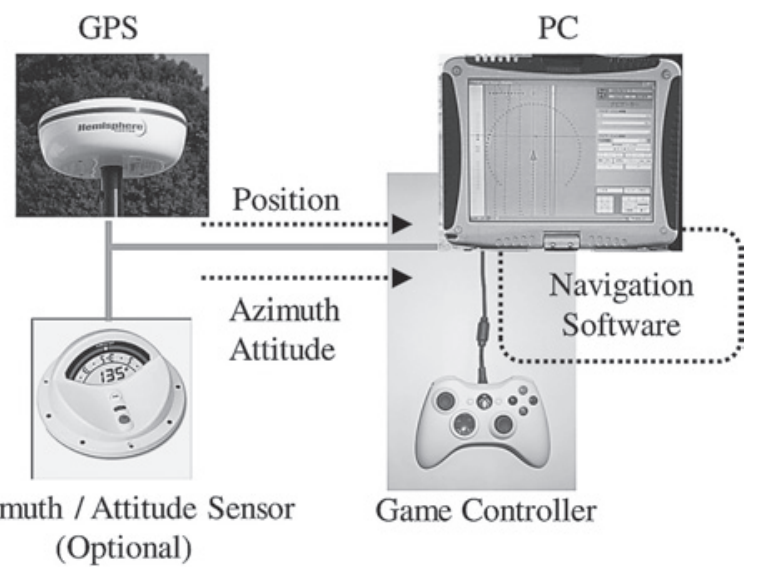

Fig.1. Composition of the navigation system

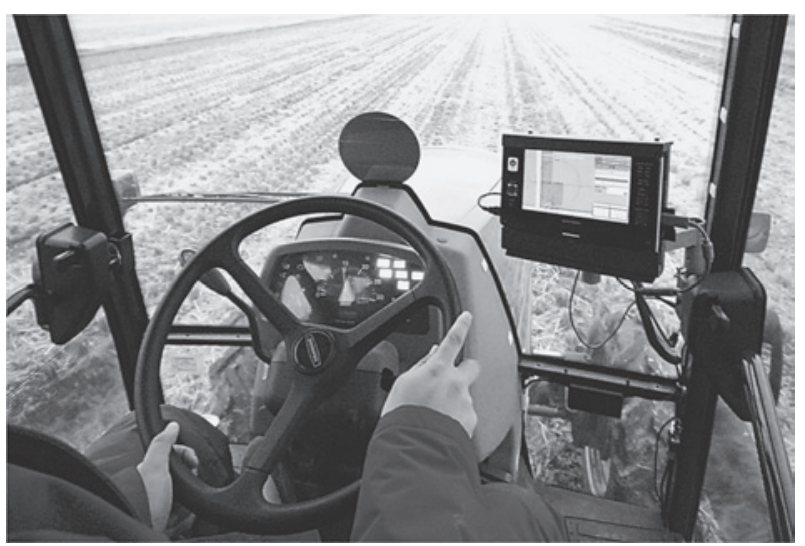

Fig.2. Navigation system mounted on a tractor sensors, control the communications ports, map information about the fields and work traces, calculate work path information for display, and plot field boundaries. These functions are controlled by software modules. The framework manages these modules and exchanges information between them. Multiple combinations of modules can be set up and are selectable when work starts, so there is no need to change settings between vehicles.

The framework also supports information on position, field boundaries, the status of the vehicle, and work traces, by exporting it to other programs such as spreadsheet software, graphics software, word processors, and geographic information systems (GIS).

The user interface controls the internal processor and displays information provided by it. It gives the highest priority to responding to user commands, and operates smoothly when enlarging, contracting, or rotating map information without causing the operator to wait.

The functions of both sections can be expanded by installing software plug-ins. The Bio-oriented Technology Research Advancement Institution (BRAIN) has developed plug-ins that (a) support precision farming by generating yield maps when connected to a combine harvester that can measure the yield and moisture content, and by varying the rate of fertilizer application according to the current position in the field when connected to a variable fertilizer applicator; and (b) steer a vehicle automatically on the basis of sensor information.

\section{(2) Screen display of navigation software}

The navigation computer displays a menu screen, a navigation screen, and a settings screen.

The menu screen is used for selecting the functions to perform and displaying the navigation screen. Work is started via the remote control or the touch panel on the PC, and the settings screen is displayed for setting input/ output conditions to and from external devices, reading map information, and so on. When plug-ins are added, newly added options are displayed.

The navigation screen (Fig. 3) is displayed during work. The map section (on the left) displays map information, including the current position of the vehicle, running and work traces, field boundaries, and the recommended path. The indicator section (on the right) displays work information, including the current state of the vehicle and implement, and permits adjustment of settings such as the work width.

\section{Using the navigation system}

The navigation system determines the work area from the current position, and displays the recommended work path in a grid based on the field boundaries and the preset work width. 


\section{(1) Registration of field boundaries}

Field boundaries can be set either from a vehicle fitted with the navigation system while it is driven around the perimeter or by walking around the perimeter with a GPS receiver and PC running the navigation system (Fig. 4). If the navigation system is mounted on a vehicle without an implement, a standard position on the vehicle can be set, and the boundaries of each field can be set accurately. Alternatively, the latitude and longitude of the field boundary can be read from a GIS file or can be set from spreadsheet software.

\section{(2) Work}

The work width is set before work starts. Parameters used in setting the work width include the maximum work width, minimum work width, and implement width. When there are no changes from the previous job, there is no need to reset the parameters.

When the vehicle enters a field, the navigation system automatically retrieves the boundaries and calculates the circumscribing rectangles of the boundaries from the reference direction of the field. It determines the optimum work width from these rectangles and the preset work width and displays the recommended path, which is based on various conditions. For example, if the width of the implement exceeds the work width (like a rotary tiller), an allowance is made for obstacles on the perimeter of the field to prevent the implement from going out of the boundaries.

The work navigation screen (Fig. 5) displays the current position and the work width, running and work traces, the reference position from which deviations are calculated, the travel direction, the reference lines for recommended paths in the travel direction and the lateral direction, the direction of the vehicle relative to the rec-

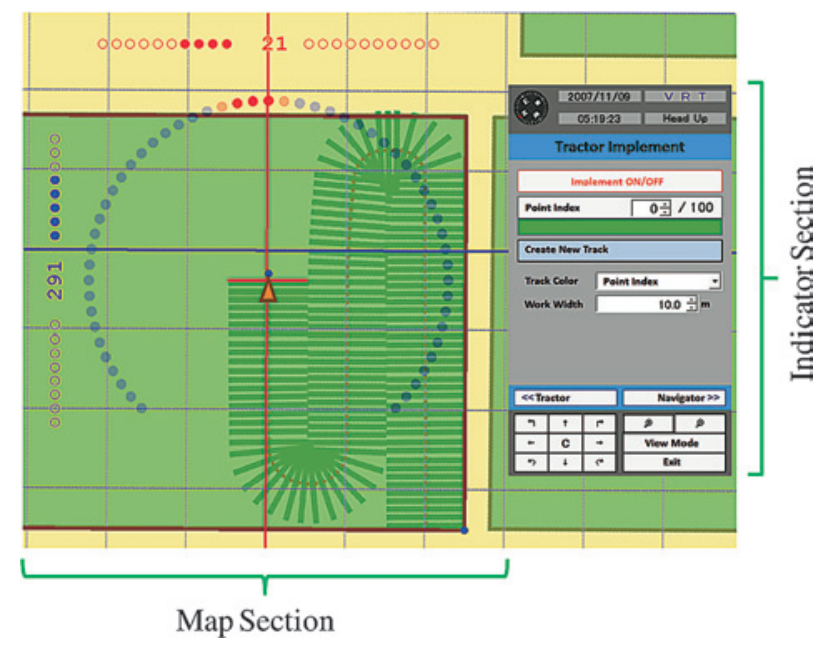

Fig.3. Navigation Screen ommended path, the deviation in the lateral direction, and the recommended steering angle. A reference line in the lateral direction is used to confirm the position at which to enter the next path.

The current position is set to the center of the implement work width and is calculated as

$$
\begin{aligned}
& P_{c}=P_{G P S}-R P_{i 2 G} \\
& P_{c}=\left(\begin{array}{l}
X_{c} \\
Y_{c} \\
Z_{c}
\end{array}\right) \\
& P_{G P S}=\left(\begin{array}{l}
X_{G P S} \\
Y_{G P S} \\
Z_{G P S}
\end{array}\right) \\
& \left.P_{i 2 G}=\left(\begin{array}{l}
x_{i 2 G} \\
y_{i 2 G} \\
z_{i 2 G}
\end{array}\right) \quad \begin{array}{ccc}
\cos \phi \cos \theta \\
\cos \psi \sin \phi+\cos \phi \sin \theta \sin \psi & \cos \phi \cos \psi-\sin \phi \sin \theta \sin \psi & -\cos \theta \sin \psi \\
\sin \phi \sin \psi-\cos \phi \cos \psi \sin \theta & \cos \psi \sin \phi \sin \theta+\cos \phi \sin \psi & \cos \theta \cos \psi
\end{array}\right)
\end{aligned}
$$

where $P_{\mathrm{c}}$ is the current position in the global coordinate system; $P_{\mathrm{GPS}}$ is the GPS-measured position in the global coordinate system; $P_{i 2 \mathrm{G}}$ is the distance from the center of the work width of the implement to the GPS antenna in the vehicle coordinate system (the origin is set to $P_{c}$, and the travel direction is defined as the $y$-axis); $R$ is the Euler angle rotation matrix of the vehicle attitude, $\varphi$ is the pitch angle, $\theta$ is the roll angle, and $\psi$ is the heading angle. Roll and pitch come from the optional attitude sensor. If the sensor is not fitted, the angles are set to zero. The heading angle is automatically taken from the azimuth sensor if it is fitted, from the GPS-measured heading, or from the heading calculated from the GPS-measured position.

The reference position from which deviations are calculated is forecast after a fixed $1.5 \mathrm{~s}$ to cancel out the

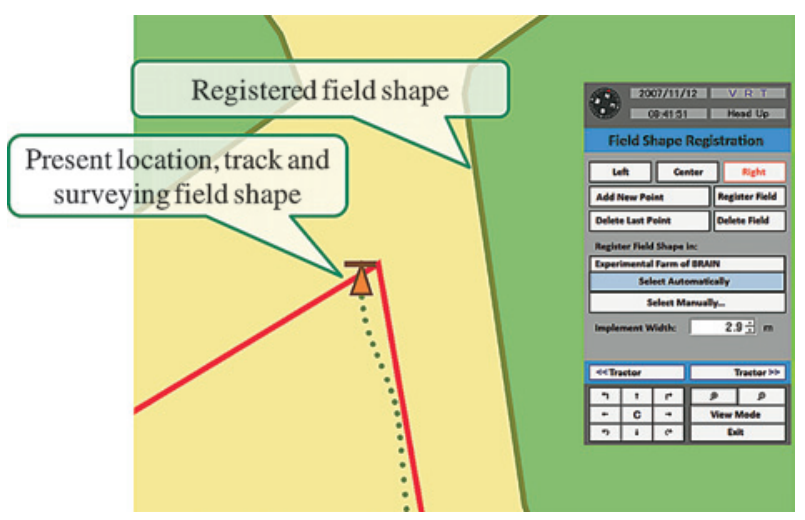

Fig.4. Example of a field shape registration view 
measuring delay of the GPS sensor or the steering delay of the operator, as

$$
\begin{aligned}
& P_{r e f}=P_{c}+v t \\
& P_{r e f}=\left(\begin{array}{c}
X_{r e f} \\
Y_{r e f} \\
Z_{r e f}
\end{array}\right) \\
& v=\left(\begin{array}{c}
X_{v} \\
Y_{v} \\
Z_{v}
\end{array}\right)
\end{aligned}
$$

where $P_{\text {ref }}$ is the reference position, $v$ is the velocity of the vehicle, and $t$ is the delay.

The deviation in the vehicle coordinate system is calculated as

$$
\begin{aligned}
& D=R^{-1}\left(P_{\text {ref }}-P_{\text {navi }}\right) \\
& D=\left(\begin{array}{l}
x_{\text {dev }} \\
y_{\text {dev }} \\
z_{\text {dev }}
\end{array}\right) \\
& P_{\text {navi }}=\left(\begin{array}{l}
X_{\text {navi }} \\
Y_{\text {navi }} \\
Z_{\text {navi }}
\end{array}\right)
\end{aligned}
$$

where $P_{\text {navi }}$ is a navigation reference point in the global coordinate system that is the nearest point of intersection between the reference lines for recommended paths in the travel direction and the lateral direction; and $D$ is the deviation from the navigation reference point in the vehicle coordinate system.

The recommended steering angle $(S)$ is calculated

$$
\left\{\begin{array}{l}
S=S_{\min }\left(\alpha x_{d e v}<S_{\min }\right) \\
S=\alpha x_{d e v}\left(S_{\min } \leqq \alpha x_{d e v} \leqq S_{\max }\right) \\
S=S_{\max }\left(\alpha x_{d e v}<S_{\max }\right)
\end{array}\right.
$$

where $S_{\min }$ is the minimum steering angle, $S_{\max }$ is the maximum steering angle, and $\alpha$ is the steering gain.

\section{Field tests}

\section{Method}

To verify the effectiveness of the navigation system, we simulated fertilizer application using a Yanmar AF650 tractor (36.8 kW) in a harvested paddy field on an experimental farm of BRAIN in Kounosu city of Saitama prefecture and evaluated the work accuracy and efficiency. We fitted a Hemisphere VectorPro navigation sensor, which can detect position (horizontal accuracy $<1 \mathrm{~m}$, $95 \%$ of the time), azimuth, and roll and pitch. The display PC was a Panasonic ToughBook CF-18. We tested combinations of operator skill (skilled/unskilled), running speed (low/high speed), and path guiding method (navigation system or by eye), as listed in Table 1.

We evaluated the work accuracy by measuring the work trace with an RTK-GPS receiver (Trimble SPS-750; horizontal accuracy: $1 \mathrm{~cm}$; vertical accuracy: $2 \mathrm{~cm}$ ) and calculating the deviation from the reference path interval (10 $\mathrm{m})$ and the ratios between missed and overlapped areas in the straight sections of all 10 paths (a total of $80 \mathrm{~m}$ ). The efficiency was calculated from the average running speed and the average interval between paths. In the sections with guide poles installed, the time required for in-

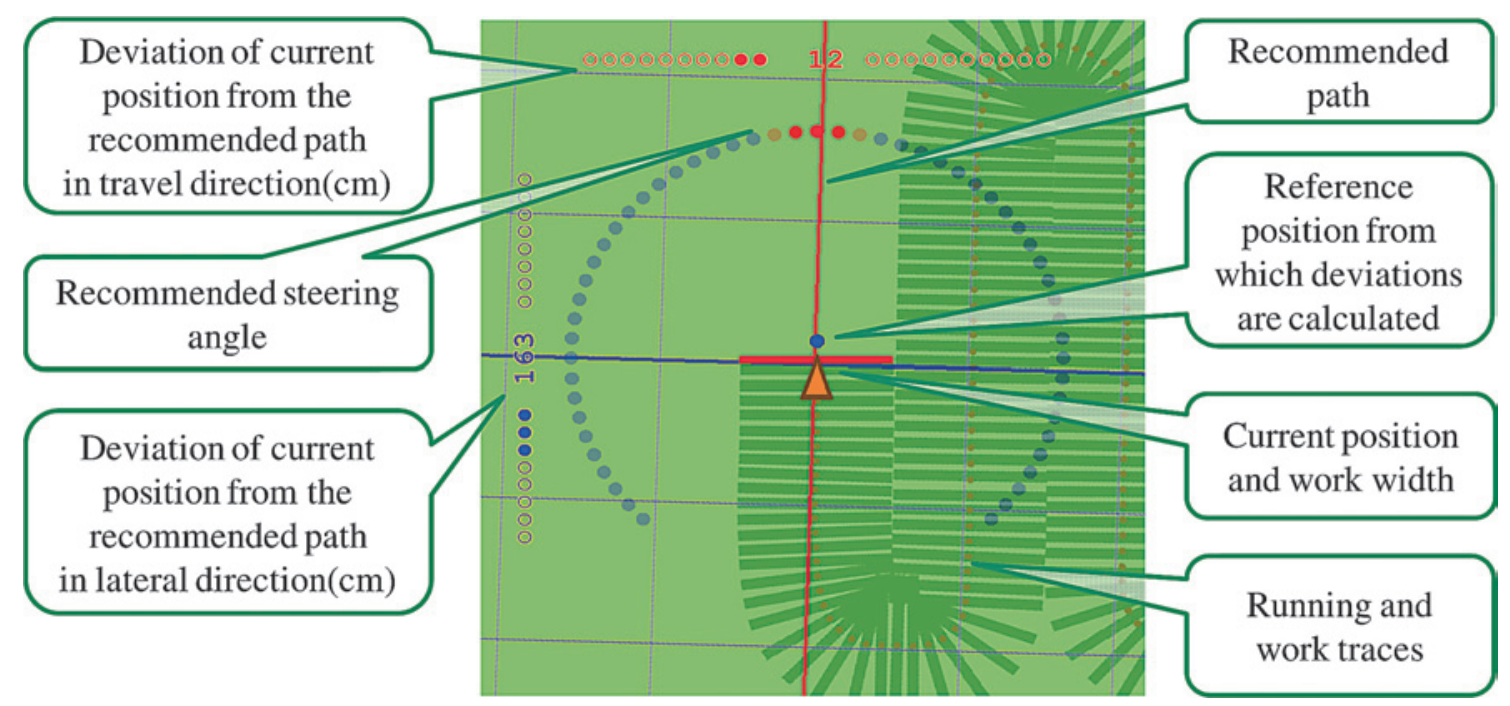

Fig.5. Navigation screen in work (map section) 
stalling and removing the poles was included in the work efficiency calculations.

\section{Results}

The test results are presented in Table 2 and Fig. 6 . Omitting guide poles greatly improved the work accura- cy. The navigation system achieved work accuracy equal to that attained with guide poles, and when the time required for installing and removing guide poles is considered, the work efficiency of the navigation system is greatly improved. There was no major difference between skilled and unskilled operators using the naviga-

Table 1. Conditions of field work tests

\begin{tabular}{lll}
\hline \hline \multicolumn{1}{l}{ Conditions } & & Outline \\
\hline Kind of work & & Simulated fertilizing work with intervals of $10 \mathrm{~m}$ between paths. \\
\hdashline Operator Skill & $\begin{array}{l}\text { Skilled } \\
\text { Unskilled }\end{array}$ & $\begin{array}{l}\text { A farming operation specialist of BRAIN } \\
\text { BRAIN researcher }\end{array}$ \\
\cline { 1 - 2 } Speed & Low & Reference speed is $1 \mathrm{~m} / \mathrm{s}$. \\
& High & Reference speed is $2 \mathrm{~m} / \mathrm{s}$. \\
\hline \multirow{2}{*}{ Guiding Method } & Navigator & Use navigation system. \\
& Guide Pole & Install guide poles (surveying pole and pile for tunnel mulch) at start/end point of paths before work. \\
& No Guide & No navigation system or guide poles used. \\
\hline
\end{tabular}

Table 2. Results of field work tests

\begin{tabular}{|c|c|c|c|c|c|c|c|c|c|}
\hline \multirow[t]{3}{*}{ Operator } & \multirow[t]{3}{*}{ Speed } & \multirow{3}{*}{$\begin{array}{l}\text { Guidance } \\
\text { Methods }\end{array}$} & \multirow{3}{*}{$\begin{array}{l}\text { Average } \\
\text { Speed } \\
(\mathrm{m} / \mathrm{s})\end{array}$} & \multicolumn{2}{|c|}{ Path Interval } & \multirow{3}{*}{$\begin{array}{c}\text { Missed } \\
\text { Area } \\
\text { Percentage } \\
\text { (\%) }\end{array}$} & \multirow{3}{*}{$\begin{array}{l}\text { Overlapped } \\
\text { Area } \\
\text { Percentage } \\
\text { (\%) }\end{array}$} & \multirow{3}{*}{$\begin{array}{l}\text { Missed / } \\
\text { Overlapped Area } \\
\text { Percentage } \\
\text { (\%) }\end{array}$} & \multirow{3}{*}{$\begin{array}{c}\text { Work } \\
\text { Efficiency } \\
\text { (ha/h) }\end{array}$} \\
\hline & & & & Average & SD & & & & \\
\hline & & & & (m) & (m) & & & & \\
\hline \multirow[t]{6}{*}{ Skilled } & \multirow[t]{3}{*}{ Low } & Navigator & 1.10 & 9.99 & 0.24 & 0.8 & 0.9 & 1.7 & 4.0 \\
\hline & & Guide Poles & 1.10 & 9.97 & 0.21 & 0.6 & 0.9 & 1.5 & 2.0 \\
\hline & & No Guide & 1.09 & 9.57 & 0.51 & 0.1 & 4.0 & 4.1 & 3.8 \\
\hline & \multirow[t]{3}{*}{ High } & Navigator & 2.06 & 9.98 & 0.35 & 1.2 & 1.4 & 2.5 & 7.4 \\
\hline & & Guide Poles & 2.04 & 9.98 & 0.27 & 0.9 & 1.1 & 2.1 & 2.7 \\
\hline & & No Guide & 2.07 & 10.12 & 0.99 & 3.8 & 2.7 & 6.4 & 7.5 \\
\hline \multirow[t]{4}{*}{ Unskilled } & \multirow[t]{2}{*}{ Low } & Navigator & 1.11 & 10.01 & 0.28 & 1.0 & 0.9 & 1.9 & 4.0 \\
\hline & & No Guide & 1.10 & 9.36 & 1.20 & 2.0 & 7.8 & 9.8 & 3.7 \\
\hline & \multirow[t]{2}{*}{ High } & Navigator & 2.06 & 10.01 & 0.32 & 1.2 & 1.1 & 2.2 & 7.4 \\
\hline & & No Guide & 2.07 & 10.65 & 0.98 & 6.8 & 1.0 & 7.8 & 7.9 \\
\hline
\end{tabular}

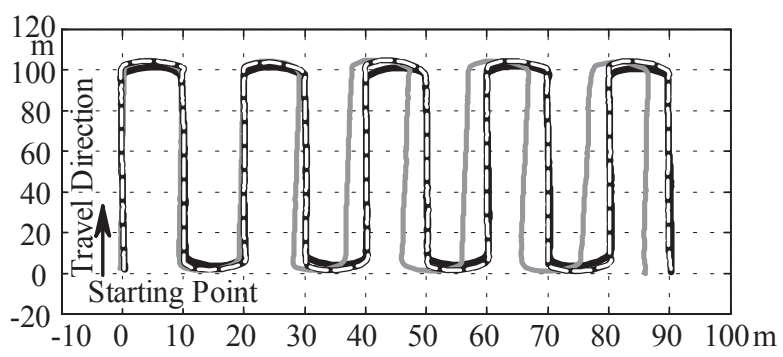

(a) Skilled Operator (Low Speed)

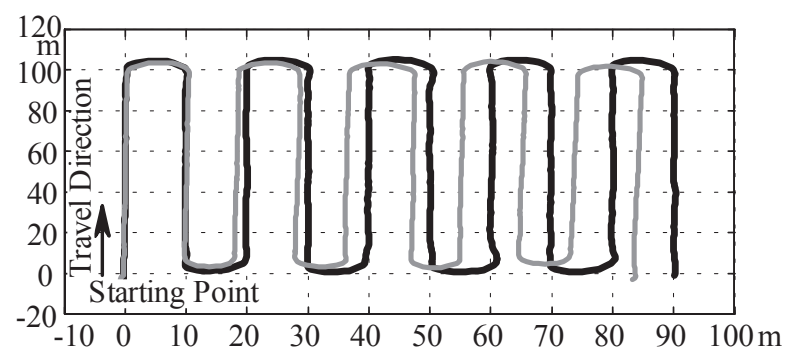

(b) Unskilled Operator (Low Speed)

Fig.6. Example of vehicle path in field work tests 
tion system. These results confirm that the navigation system improves work accuracy and efficiency.

\section{Field evaluation}

\section{Method}

We used the navigation system for broadcast-fertilizing, rotary tilling, and puddling of flooded paddy fields on a paddy-rice farm in Higashikawa Town, Kamikawa County, Hokkaido, from April 2006 to May 2007. We evaluated the ease of use and improved the software. We also used it for puddling and topdressing in a paddy field at the experimental farm of BRAIN from May to August 2007 and evaluated its ease of use.

\section{Results}

The navigation system operated stably and proved easy to use. Farmers found it easy to handle, although they needed some practice first. Because field navigation is useful in various types of work, they hoped that it would be put on the market soon. It improved efficiency by allowing guide poles to be eliminated. Through these tests, we were able to improve the software to make it easier to recognize the recommended path on the screen. We added new functions to automatically determine the work field from the current position and to adjust the work width automatically so that the optimum interval between paths can be maintained.

\section{Conclusion}

We developed a navigation system for agricultural vehicles to support precision farming and to eliminate time loss and needless operation, and confirmed its effectiveness in the field. It improved work accuracy and efficiency by eliminating the need for installing guide poles and could be operated by unskilled workers. Monitoring tests confirmed that it could operate stably over a long period and was easy to use.

Timber Tech (http://www.timber.co.jp) and GNSS Technologies (http://www.gnss.co.jp) initiated trial sales of the navigation system in 2007.

We anticipate that the navigation system will play an important role in achieving the inconsistent objectives of improving productivity and food safety while reducing environmental impacts. We intend to further refine it in the future.

\section{References}

1. Gotoh, T., Makino, E. \& Hayashi, K. (2000) Investigation of Precision Farming Technology in North America. Biooriented Technology Research Advancement Institution, Saitama, Japan, 23-24 [In Japanese].

2. Hamada, Y., Matsuo, Y. \& Ogura, A. (2004) Software framework for agricultural vehicle navigation. In Automation Technology for Off-Road Equipment 2004, eds. Zhang, Q., Iida, M. \& Mizushima, A., ASAE, St. Joseph, MI, USA, 283-291.

3. Hirota, K. (2008) GPS utilization trend for agricultural applications. Nogyokikai gakkaishi (J. JSAM), 70(1), 29-33 [In Japanese].

4. Trimble Navigation Limited, (2007) Trimble SPSx50 and SPSx51 Modular GPS Receiver, Trimble Navigation Limited, Sunnyvale, CA, USA, 111-128. 